\title{
Pleomorphic Xanthoastrocytoma, Anaplastic Pleomorphic Xanthoastrocytoma, and Epithelioid Glioblastoma: Case Series with Clinical Characteristics, Molecular Features and Progression Relationship
}

\section{Zhiying Lin}

Southern Medical University Nanfang Hospital

\section{Runwei Yang}

Southern Medical University Nanfang Hospital

\section{Haojie Zheng}

Southern Medical University Nanfang Hospital

\section{Zhiyong Li}

Southern Medical University Nanfang Hospital

Guozhong Yi

Southern Medical University Nanfang Hospital

Huixia Han

Southern Medical University Nanfang Hospital

Qianchao Wu

Genomicare Biotechology (Shanghai) Co.Ltd.,Shanghai,China

\section{Songtao Qi}

Southern Medical University Nanfang Hospital

Guanglong Huang ( $\nabla$ hgl1020@163.com)

Southern Medical University Nanfang Hospital https://orcid.org/0000-0002-1589-9202

\section{Research}

Keywords: pleomorphic xanthoastrocytoma, anaplastic pleomorphic xanthoastrocytoma, epithelioid glioblastoma, clinical characteristics, molecular features, progression relationship

Posted Date: April 20th, 2021

DOl: https://doi.org/10.21203/rs.3.rs-99690/v2 
License: (c) (i) This work is licensed under a Creative Commons Attribution 4.0 International License. Read Full License 


\section{Abstract}

Background: Pleomorphic xanthoastrocytoma (PXA), anaplastic pleomorphic xanthoastrocytoma (APXA), and epithelioid glioblastoma (E-GBM) show overlapping features. However, little is known about their clinical characteristics, molecular features and relationship with progression.

Methods: Fourteen patients diagnosed at Nanfang Hospital from 2016 to 2019 were enrolled, including eleven PXA patients, two A-PXA patients, and one E-GBM patient. All tumour tissue samples of the fourteen patients were examined by immunohistochemical staining (MGMT, VEGF, BRAF-V600E, etc.). The recurrent tumour tissue of the patient with E-GBM arising from A-PXA was screened to detect 11 glioma markers (MGMT, BRAF-V600E, etc.) and chromosome 1p/19q by next-generation sequencing (NGS).

Results: The mean age of 13 patients with PXA or A-PXA was 25.4 years; twelve of these patients had tumours at supratentorial regions. VEGF positivity was detected in the tumour samples of 13 patients, MGMT positivity in 10 patients, and BRAF-V600E positivity in 7 patients. For the tumour sample of the EGBM patient who survived for up to 10 years after the fourth resection, BRAF V600E was wild type in the sample obtained from the first surgery, while it was mutant in the second, third, and fourth surgeries. In contrast, the promoter status of MGMT in the four surgeries was unmethylated. The NGS results showed that the mutation frequencies of BRAF V600E in the second, third and fourth surgeries were $14.06 \%$, $9.13 \%$ and $48.29 \%$, respectively.

Conclusions: Collectively, the results suggest that patients with A-PXA may relapse multiple times and eventually progress to E-GBM with the BRAF-V600E mutation.

\section{Introduction}

Pleomorphic xanthoastrocytoma (PXA) is a low-grade brain tumour (WHO II) that was first reported by Kepes et al in 1979 [1]. PXA accounts for less than 1\% of all brain tumours [2], and most of the tumours are located in the supratentorial compartment, mainly in the temporal lobe, followed by the frontal lobe and the parietal lobe [3]. On imaging, these tumours show solid enhanced nodules, often accompanied by eccentric peripheral cystic components and peripheral oedema [4]. Histologically, these pleomorphic tumours show different characteristics: spindle cells, multinucleated and fibrous giant cells, eosinophilic granular bodies, polygonal cells and lipid-laden xanthomatous astrocytes [1, 3].

anaplastic pleomorphic xanthoastrocytoma (A-PXA) is similar to PXA in terms of signs and symptoms, but it may exhibit aggressive biological behaviour and has a relatively poorer prognosis than PXA $[5,6]$. The frequency of the BRAF V600E mutation is lower in A-PXA than in PXA and not significantly different between paediatric patients and adults, but the prognostic significance of the mutation is not clear $[2,7]$. A decisive genetic feature of PXA and anaplastic PXA is the homozygous deletion of CDKN2A combined with RAF changes [8]. PXA and A-PXA are usually seen in children and young people [1]. No specific 
epidemiological data are available regarding A-PXA compared with PXA. The acquisition of the molecular characteristics of PXA and A-PXA helps to reveal the genetic evolution in these tumours.

Epithelioid glioblastoma (E-GBM) is a rare GBM variant newly added to IDH-wild-type GBM in the 2016 WHO classification [6]. Although the BRAF V600E mutation is rarely found in GBM, it has been identified at a relatively high frequency (50\%) in E-GBM [9]. It is unclear what genetic mechanism may cause the appearance of epithelioid morphology.

We analysed the clinical and molecular characteristics of PXA/A-PXA and summarized the possible genetic evolution types of PXA and A-PXA. Then, by studying the characteristics of a patient with E-GBM arising from A-PXA after three recurrences, we speculated on the possible malignant progression of APXA. Histological and molecular analyses can be performed during the first resection and subsequent recurrences. Histologically, the E-GBM patient was diagnosed with A-PXA based on the first, second, and third surgical specimens but E-GBM based on the fourth surgical specimen. The samples from the four operations of this E-GBM patients were detected by next-generation sequencing (NGS). The results suggest that patients with A-PXA may relapse multiple times and eventually progress to E-GBM with the BRAF-V600E mutation.

\section{Materials And Methods}

\section{Case Collection and Histopathological Information}

The Institutional Review Board of Nanfang Hospital affiliated with Southern Medical University approved the study. This study is retrospective Eleven PXA patients, 2 A-PXA patients, and 1 E-GBM patient diagnosed by pathologists from 2016 to 2019 in Nanfang Hospital were enrolled. In this study, clinical data, including age, sex, pathological diagnosis (according to the 2016 WHO central nervous system tumour classification), anatomic location of glioma, tumour size, and pathological molecular markers, were collected. Three neuro-oncologists reviewed medical records for neuroimaging features and treatment options. All specimen slides were reviewed by pathologists to confirm the diagnosis of all cases. For the diagnosis of PXA, the criteria determined by Brat et al. [3] were used. All our cases satisfied the diagnostic criteria for PXA: mitotic activity of PXA was $\leq 5$ mitoses per 10 high-power fields (HPFs) [10], and the detection methods of A-PXA were based on either mitotic rate $>5 / 10$ HPFs or the presence of necrosis on histopathological findings [2, 7]. GBM displays nuclear atypia, cellular pleomorphism, mitotic activity, a diffuse growth pattern, microvascular proliferation and/or necrosis [6]. In addition, we conducted glial fibrillary acidic protein (GFAP) immunotherapy in all cases. Immunocytochemical detection of GFAP can identify the astrological nature of tumour cells and thus support the exclusion of nonglial neoplasms resembling PXA or A-PXA [11].

\section{Molecular Detection}

The pathological molecular markers mainly included GFAP (OriGene, monoclonal, 1:100 dilution), epithelial membrane antigen (EMA, OriGene, monoclonal, 1:100 dilution), 06-methylguanine-DNA 
methyltransferase (MGMT, OriGene, monoclonal, 1:100 dilution), neuronal nuclei (Neu-N, OriGene, monoclonal, 1:100 dilution), oligodendrocyte transcription factor 2 (Oligo-2, OriGene, monoclonal, 1:100 dilution), epidermal growth factor receptor (EGFR, OriGene, monoclonal, 1:100 dilution), vascular endothelial growth factor (VEGF, OriGene, polyclonal, 1:100 dilution), isocitrate dehydrogenase 1 (IDH1, OriGene, monoclonal, 1:100 dilution), Ki-67 (OriGene, monoclonal, 1:100 dilution), ATRX chromatin remodeler (ATRX, OriGene, polyclonal, 1:100 dilution), CD34 molecule (CD34, OriGene, monoclonal, 1:100 dilution), Synaptophysin (Syn, OriGene, monoclonal, 1:100 dilution), BRAF V600E (Roche, monoclonal, 1:100 dilution), H3K27M (OriGene, polyclonal, 1:100 dilution), Neurofilament (NF, OriGene, monoclonal, 1:100 dilution), Chromogranin A (CgA, OriGene, monoclonal, 1:100 dilution), CD20 (OriGene, monoclonal, 1:100 dilution), Cytokeratin (CK, OriGene, monoclonal, 1:100 dilution), CD30 (OriGene, monoclonal, 1:100 dilution), Vimentin (OriGene, monoclonal, 1:100 dilution), Capicua (CIC, OriGene, monoclonal, 1:400 dilution), far upstream element-binding protein 1 (FUBP1, OriGene, monoclonal, 1:400 dilution), Desmin (OriGene, monoclonal, 1:100 dilution) and reticular fibres (Abcam, monoclonal, 1:500 dilution).

\section{Fluorescence in situ hybridization (FISH) detection}

We performed FISH on formalin-fixed paraffin-embedded (FFPE) tissue sections using the same commercial 1p/19q probes (Vysis paired probes 1p36/1q25 and 19q13/19p13, Abbott Laboratories). In brief, 5 - $\mu \mathrm{m}$-thick formalin-fixed, paraffin-embedded sections were deparaffinized, pretreated with Vysis pretreatment solution (1 N, NaSCN, Vysis 32-801200, Abbott Molecular Inc.) and digested in protease solution (Vysis 32-801200, Abbott Molecular Inc.). The probe mix (5 to $15 \mu \mathrm{l}$ ) was added to each slide according to the manufacturer's instructions. Target DNA and probes were codenatured at $74^{\circ} \mathrm{C}$ for 5 minutes and incubated at $37^{\circ} \mathrm{C}$ overnight in a humidified hybridization chamber (ThermoBrite, Abbott Molecular Inc.). Post-hybridization washes were performed in $2 x \mathrm{SSC} / 0.3 \% \mathrm{NP}-40$ for 2 minutes at $75^{\circ} \mathrm{C}$. Finally, the slides were air-dried and counterstained with 4,6-diamidino-2-phenylindole (DAPI, Cell Signaling Technology, \#4083).

\section{Tissue Processing and NGS Panel Genotyping}

NGS panel sequencing and analysis were performed at the Genomics Laboratory of GenomicCare Biotechnology (Shanghai, China). For the FFPE tissue, DNA was extracted using the MagMAX FFPE DNA/RNA Ultra Kit (cat\# A31881, Thermo Fisher, Waltham, MA, USA) on a KingFisher Flex System (Thermo Fisher). The extracted DNA was sheared using a Covaris L220 sonicator, and then library preparation and capture were performed using a Tecan EVO 150 (Thermo Fisher), and the DNA was sequenced on an lon S5 sequencer (Thermo Fisher) to generate paired-end reads. After removing adapters and low-quality reads, the reads were aligned to the National Center for Biotechnology Information (NCBI) human genome reference assembly hg19 using the Burrows-Wheeler Aligner algorithm and further processed using the Genome Analysis Toolkit (GATK, version 3.5), including the GATK Realigner Target Creator to identify regions that needed to be realigned. Somatic single-nucleotide variants (SNVs), insertions and deletions (indels), and copy number variations (CNVs) were determined using MuTect/ANNOVAR/dbNSFP31, VarscanIndel, and CNVnator software, respectively, as reported in Zang et al. [12]. 


\section{Statistical analysis}

The SPSS statistical software package (version 25, IBM Corp.) was used for all analyses. Categorical variables are shown as numbers and percentages, while continuous variables are shown as the mean and standard deviation (SD).

\section{Results}

\section{Clinical and histopathological features of PXA/A-PXA}

A total of 13 patients who were diagnosed with PXA or A-PXA in Nanfang Hospital were utilized to analyse and summarize the clinicopathological and molecular features of PXA/A-PXA. One patient with E-GBM arising from A-PXA was used to analyse the possible disease progression process of A-PXA.

Five females and eight males were diagnosed with PXA/A-PXA. The patients were aged 7 to 53 years and included four paediatric cases. The supratentorial to subtentorial ratio was 12.00 . The mean age of the patients was 25.40 years, and the mean diameter of the tumour was $3.50 \mathrm{~cm}(\mathrm{SD}=0.48 \mathrm{~cm})$.

In our study, it was found that in all patients, GFAP and VEGF were positively expressed, chr 1p/19q was intact, and H3K27M was negatively expressed. Nine of 13 patients showed Ki-67 positivity in the range of $0-3 \%, 7$ of 13 patients had BRAF V600E mutations, 5 of 13 patients were EMA negative, and 3 of 13 patients were MGMT promoter methylated. Detailed information for this cohort of PXA and A-PXA patients is provided in Table 1.

\section{E-GBM arising from A-PXA}

To analyse the possible malignant progression of A-PXA, we enrolled a patient with E-GBM who relapsed from A-PXA in this study (Table 2). The E-GBM patient was a 40-year-old woman who visited Nanfang Hospital on November 30th, 2010, due to a headache and blurred vision for half a month. Magnetic resonance imaging (MRI) showed that the left parietal lobe occupied a space that was considered to be tumourous changes with a size of approximately $5.8 * 3.1 * 4.1 \mathrm{~cm}$ and a clear border. A reinforced nodule with a size of approximately $2.4 * 0.9 \mathrm{~cm}$ was thickened adjacent to the meninges, and the spaceoccupying effect was obvious. The left ventricle was compressed, and the midline structure was shifted to the opposite side (Fig. 1). After the first surgery, the pathological diagnosis was A-PXA. The first surgical specimen showed that the arrangement of cancer cells was compact and flaky, with transparent pronucleus cytoplasm, different cell sizes, heterogeneity, no obvious necrosis and local vascular endothelial cell proliferation (Table 3). The molecular markers were characterized by Ki-67 (label index: 10\%), GFAP (positive), MGMT (unmethylated), EMA (negative), oligarch-2 (positive), IDH1 (wild type) and BRAF V600E (wild type) (Fig. 2). FISH detection showed that chr 1p and 19q were intact.

The patient received postoperative radiotherapy. Follow-up MRI scans were carried out every year. There were no recurrences seen on MRI six years after the first operation. However, six months after the follow- 
up in June 2016, the patient experienced right limb weakness. The preoperative MRI scan suggested tumour recurrence in the left parietal lobe (Fig. 1). After the second surgery, the pathological diagnosis was A-PXA (Fig. 1). The cancer cells were densely packed, with a proliferation of small blood vessels in the interstitium and local collagen fibres. Acute inflammatory cell infiltration and mitosis were observed. Compared with the first postoperative immunohistochemical results, the second postoperative immunohistochemical results showed mostly similar molecular marker findings. BRAF V600E was mutant, and the newly added markers were Neu-N (negative), TP53 (wild-type), VEGF (positive), EGFR (positive), ATRX (wild-type), CD34 (partial positive), CD68 (positive) (Figure S1A), and reticular fibres (positive), but the Ki-67 labelling index fell to $5 \%$ (Fig. 2A). The FISH detection of 1p/19q was the same as the first postoperative result. The patient received postoperative radiotherapy and temozolomide (TMZ) chemotherapy.

Eight months after the second surgery, the patient experienced weakness of the lower extremities for more than one month. The preoperative MRI scan showed recurrent glioma at the left parietal lobe (Fig. 1). After the third surgery, the pathological diagnosis was A-PXA, WHO grade III. The size and shape of the cancer cells were different. There was hyperplasia of small blood vessels in the stroma, local haemorrhage, and no obvious necrosis. Compared with the second postoperative immunohistochemical results, the third postoperative immunohistochemical results demonstrated similarities for most markers, except that the Ki-67 index increased to $8 \%$, BRAF V600E was mutant, and Neu-N was changed from negative to residual neurons positive (Fig. 2). FISH detection showed that chr $1 p$ and $19 q$ were intact. One month after the operation, the patient received radiotherapy and TMZ chemotherapy. The dose of the first cycle of TMZ chemotherapy was $200 \mathrm{mg}\left(150 \mathrm{mg} / \mathrm{m}^{2}\right)$ orally once a day for 6 days. In the second to sixth cycles, the dose was $300 \mathrm{mg}\left(200 \mathrm{mg} / \mathrm{m}^{2}\right)$ orally once a day for 5 days for every cycle.

After two years, the patient experienced weakness in the right limb, which gradually increased for more than a month. Four limbs had involuntary movement, the left muscle strength was normal, the muscle strength grade of the right upper limb was zero, and that of the lower limb was IV. A preoperative MRI head perfusion enhanced scan indicated a left frontal-parietal lobe occupation of $2.0 \mathrm{~cm} \times 1.2 \mathrm{~cm}$, considering the possibility of tumour recurrence (Fig. 1). After the fourth surgery, the pathological diagnosis was E-GBM, WHO grade IV, IDH-1 wild-type. The tissue from the fourth surgery was densely packed with cancer cells, small blood vessels and palisade necrosis. Compared with the third postoperative immunohistochemical results, the fourth postoperative immunohistochemical results showed similar findings for GFAP, MGMT, EMA, IDH1, Oligo-2, EGFR, BRAF V600E, TP53, and ATRX. The newly added markers were CIC (wild-type), FUBP1 (positive), and H3K27M (negative expression) (Figure S1B). The Ki-67 index increased to $35 \%$, Neu-N was changed from residual neurons positive to negative, and VEGF changed from positive expression to negative expression (Fig. 2B). FISH detection showed that chr $1 p$ and $19 q$ were intact. Postoperative radiotherapy and a TMZ-intensive regimen were considered as adjuvant chemotherapy. The dose of the first cycle of TMZ chemotherapy was $200 \mathrm{mg}\left(150 \mathrm{mg} / \mathrm{m}^{2}\right)$ orally once a day for 6 days. In the second to sixth cycles, the dose was $300 \mathrm{mg}\left(200 \mathrm{mg} / \mathrm{m}^{2}\right)$ orally once a day for 5 days for every cycle. Detailed information on the pathological characteristics and molecular 
features of the E-GBM patient is provided in Supplementary Table 3 and Supplementary Table 4, respectively.

\section{NGS panel analysis}

We then performed NGS on the E-GBM and A-PXA specimens from this patient. The detected gliomarelated markers and chromosomes were IDH1, IDH2, ATRX, TERT, TP53, H3F3A, MGMT, BRAF, EGFR, PIK3CA, HIST1H3B, and chr 1p/19q (Supplementary Table 5). The results indicated that the mutation frequencies of BRAF-V600E in the second, third, and fourth specimens were $14.06 \%, 9.13 \%$, and $48.29 \%$, respectively. IDH1, IDH2, ATRX, TERT, TP53, H3F3A, HIST1H3B, MGMT, EGFR, and PIK3CA were wild-type, and chr $1 \mathrm{p} / 19 \mathrm{q}$ was intact.

\section{Discussion}

PXA and A-PXA are rare forms of glioma, which is why the number of cases in our study is limited. In most of our cases, A-PXA was removed for the first time. A-PXA may occur during the initial resection, with anaplastic characteristics, or develop from PXA to A-PXA, but the prognosis of both is usually poor [13-15].

PXA has a favourable prognosis, with a 10-year overall survival rate of approximately $70 \%[11,16,17]$. However, $5 \%-20 \%$ of patients with PXA experience recurrence with malignant transformation. PXA rarely transforms into anaplastic pleomorphic xanthoastrocytoma (A-PXA) or glioblastoma (GBM) [18]. Phillips et al. reported that a decisive genetic feature of PXA and anaplastic PXA is the homozygous deletion of CDKN2A combined with RAF changes [8].

E-GBM is similar to A-PXA and exhibits epithelioid characteristics. A-PXA should be strictly distinguished from GBM [19]. At the histological and molecular levels, A-PXA may be difficult to distinguish from E-GBM because the two tumour types usually show BRAF V600E mutations. E-GBM may coexist with PXA [20], and a case report of E-GBM arising from PXA has recently been reported, but the relationship between EGBM and the malignant progression of PXA needs further clarification [21]. EZH2 expression and BRAF V600E mutations might be helpful to evaluate the prognoses of E-GBM and A-PXA patients [22].

In our research on the clinical characteristics and molecular features of PXA and A-PXA, we found that $53.85 \%(7 / 13)$ of the patients were confirmed to have BRAF V600E mutations, VEGF was positively expressed, H3K27M was negatively expressed, and $85.71 \%$ (6/7) of patients were CD34 positive. Similar to that in previous studies [23, 24], BRAF V600E mutations occurred in approximately $60 \%$ of PXA cases. There is currently no literature about the relationship between PXA/A-PXA and VEGF. In our study, all PXA/A-PXA cases expressed VEGF, which may be the common genetic evolution of PXA/A-PXA.

We studied the possible malignant progression of A-PXA through a case of E-GBM recurring from A-PXA. We described the case of E-GBM developing at the site of a previously resected A-PXA with the BRAF V600E mutation 10 years after the initial surgery. Histologically, E-GBM was reported to possess reticulin, similar to the present case, which may prompt the diagnostic consideration of A-PXA. However, E-GBM 
showed more cytologically uniform cells and a lack of eosinophilic granular bodies [2]. In addition, the clinical course of this case was consistent with that of E-GBM tumours, including invasive progression and cranial spinal cord dissemination [13-15, 25]. A-PXA and E-GBM are similar in histology, immunohistochemistry, and molecular and clinical characteristics. Although PXA rarely transforms into APXA or GBM [18], there have been some related studies of PXA transformation into GBM so far. For example, 1) a case of secondary disease with an IDH1 mutation has been reported, which is a common type of molecular feature of secondary GBM secondary to astrocyte tumours [2]. 2) Shingo Tanaka et al. described a case of E-GBM developing at the site of a previously resected PXA with the BRAF V600E mutation 13 years after the initial surgery [21]. However, to our knowledge, this is the first report on E-GBM caused by A-PXA.

For this case of E-GBM, histological studies and molecular analyses were performed for the primary resection and three relapse specimens (Fig. 3). Through immunohistochemical analysis, it was found that IDH1 was wild-type, the MGMT promoter was unmethylated, BRAF V600E was wild-type (tissue from first resection) and mutated (tissues from second, third, and fourth resections), and VEGF was positive (tissues from second and third resections) and negative (tissue from fourth resection). To identify the genetic evolution of E-GBM, we specifically performed NGS panel tests on tumour tissues from the four resection. According to the results of the NGS panel, total of 11 glioma markers and chr 1p/19q were detected in the E-GBM patient, and the results indicated that BRAF V600E was mutated in only the second, third, and fourth surgeries.

IDH1 and TP53 mutations are usually not detected in A-PXA and E-GBM [15, 25-30]. The comprehensive immunohistochemical and NGS panel results showed that our case did not contain mutations in these genes in A-PXA and E-GBM, indicating that these genes did not affect the progression from A-PXA to EGBM. In the case reported by Shingo Tanaka et al. [21], the MGMT promoter was positive in E-GBM but not in PXA. It was speculated that during the transformation from PXA to E-GBM, the state of the MGMT promoter changed from methylated to unmethylated. In contrast, in our case, the MGMT promoters in APXA and E-GBM were unmethylated, and only $23.08 \%$ of the 13 patients with PXA/A-PXA showed MGMT promoter methylation. In this research, we found that VEGF in E-GBM patients was positive (second and third resection) and negative (fourth resection), and the VEGF levels of the 13 patients with PXA/A-PXA in this study were positive. It may be possible that the status of VEGF changed from positive expression to negative expression with the transformation from A-PXA to E-GBM. Further analysis is needed to study whether the genomic level changes during the transformation from A-PXA to E-GBM.

Collectively, this study investigated the possible common clinical pathological and molecular characteristics of PXA and A-PXA. This case study suggests that E-GBM may result from the malignant transformation of A-PXA. To the best of our knowledge, this is the first report of E-GBM arising from APXA. To clarify the relationship between A-PXA and E-GBM, it is necessary to study more cases.

\section{Abbreviations}


PXA (pleomorphic xanthoastrocytoma); A-PXA (anaplastic pleomorphic xanthoastrocytoma); E-GBM (epithelial glioblastoma); GBM (glioblastoma); WES (whole exome sequencing); WHO (World Health Organization); NGS (next-generation sequencing); GBM (glioblastoma); CNS (central nervous system); NCBI (National Center for Biotechnology Information); SNV (somatic single-nucleotide variant); CNV (copy number variation); IDH1 (isocitrate dehydrogenase 1); IDH-wt (IDH-wild type); GFAP (glial fibrillary acidic protein); FFPE (formalin-fixed paraffin-embedded); EMA (epithelial membrane antigen); MGMT (O6methylguanine-DNA methyltransferase); Neu-N binding (neuronal nuclei); Oligo-2 (oligodendrocyte transcription factor 2); EGFR (epithelial growth factor receptor); VEGF (vascular endothelial growth factor); CIC (epidermal growth factor receptor); FUBP1 (far upstream element binding).

\section{Declarations}

\section{Acknowledgements}

A particular acknowledgement to the medical staff involved in the study. We want to express our thank to Haiyan Xu, Yajuan Wang, Chao Chen, Junlu Liu, and Yawei Liu for their help during the peer-review process.

\section{Funding}

This study was supported by the Guangdong Science and Technology Department (2016A040403053).

\section{Availability of data and material}

The data analysed in this study can be accessed by sending a request to the corresponding author.

\section{Author information}

\section{Affiliations}

Department of Neurosurgery, Nanfang Hospital, Southern Medical University, Guangzhou 510515, Guangdong, China

Zhiying Lin; Runwei Yang; Haojie Zheng; Haiyan Xu; Zhiyong Li; Guozhong Yi; Huixia Han; Qianchao Wu; Songtao Qi; Yajuan Wang; Chao Chen; Junlu Liu; Yawei Liu; Xiaoyan Wang; Guanglong Huang

The Laboratory for Precision Neurosurgery, Nanfang Hospital, Southern Medical University, Guangzhou 510515, Guangdong, China

Zhiying Lin; Runwei Yang; Haojie Zheng; Haiyan Xu; Zhiyong Li; Guozhong Yi; Huixia Han; Qianchao Wu; Songtao Qi; Yajuan Wang; Chao Chen; Junlu Liu; Yawei Liu; Xiaoyan Wang; Guanglong Huang

\section{Nanfang Glioma Center, Guangzhou 510515, Guangdong, China}

Guozhong Yi; Zhiyong Li; Xian Zhang; Songtao Qi; Guanglong Huang 
Department of Pathology, Nanfang Hospital, Southern Medical University, Guangzhou 510515, Guangdong, China

Huixia Han

Genomicare Biotechnology (Shanghai) Co. Ltd., Shanghai, China

Qianchao Wu

\section{Contributions}

Conception and design of the work: GLH and YWL. Acquisition, analysis and interpretation of the data: ZYL, RWY, HJZ, HYX, ZYL, GZY, HXH, QCW, STQ, and GLH. Drafting of the manuscript: ZYL and GLH. Critical revision for important intellectual content: ZYL, RWY, GZY, ZYL, STQ, and GLH. All authors approved the final version of this manuscript.

\section{Corresponding authors}

Correspondence to Guanglong Huang

\section{Ethics declarations}

\section{Ethics approval and consent to participate}

Written consent was obtained from a patient or legal guardian on behalf of the participants under the age of 16. The Institutional Review Board of Nanfang Hospital affiliated with Southern Medical University approved the study. The ethical committee approval number is NFGC-001-MCP, and the date of approval is December 18, 2013.

\section{Consent for publication}

Written informed consent was obtained from the patients for the publication of this case report. All the authors listed have approved the enclosed manuscript.

\section{Competing interests}

The authors declare that they have no competing interests.

\section{References}

1. Kepes JJ, Rubinstein LJ, Eng LF. Pleomorphic xanthoastrocytoma: a distinctive meningocerebral glioma of young subjects with relatively favorable prognosis. A study of 12 cases. Cancer. 1979;44:1839-52.

2. Ida CM, Rodriguez FJ, Burger PC, Caron AA, Jenkins SM, Spears GM, Aranguren DL, Lachance DH, Giannini C. Pleomorphic Xanthoastrocytoma: Natural History and Long-Term Follow-Up. Brain 
Pathol. 2015;25:575-86.

3. Giannini C, Scheithauer BW, Burger PC, Brat DJ, Wollan PC, Lach B, O'Neill BP. Pleomorphic xanthoastrocytoma: what do we really know about it? Cancer 1999, 85:2033-2045.

4. Rippe DJ, Boyko OB, Radi M, Worth R, Fuller GN. MRI of temporal lobe pleomorphic xanthoastrocytoma. J Comput Assist Tomogr. 1992;16:856-9.

5. Fukushima H, Nakano Y, Ishii N, Nozuchi N, Okuno T, Yamasaki K, Okada K, Fujisaki H, Kunihiro N, Matsusaka $Y$, et al. Histological and genetic analysis of anaplastic pleomorphic xanthoastrocytoma suspected of malignant progression over a 12-year clinical course. Pathol Int. 2019;69:608-13.

6. Louis DN, Perry A, Reifenberger G, von Deimling A, Figarella-Branger D, Cavenee WK, Ohgaki H, Wiestler OD, Kleihues P, Ellison DW. The 2016 World Health Organization Classification of Tumors of the Central Nervous System: a summary. Acta Neuropathol. 2016;131:803-20.

7. Schmidt Y, Kleinschmidt-DeMasters BK, Aisner DL, Lillehei KO, Damek D. Anaplastic PXA in adults: case series with clinicopathologic and molecular features. J Neurooncol. 2013;111:59-69.

8. Phillips JJ, Gong H, Chen K, Joseph NM, van Ziffle J, Bastian BC, Grenert JP, Kline CN, Mueller S, Banerjee $A$, et al. The genetic landscape of anaplastic pleomorphic xanthoastrocytoma. Brain Pathol. 2019;29:85-96.

9. Kleinschmidt-DeMasters BK, Aisner DL, Birks DK, Foreman NK. Epithelioid GBMs show a high percentage of BRAF V600E mutation. Am J Surg Pathol. 2013;37:685-98.

10. Villa C, Miquel C, Mosses D, Bernier M, Di Stefano AL. The 2016 World Health Organization classification of tumours of the central nervous system. Presse Med. 2018;47:e187-200.

11. Gelpi E, Popovic M, Preusser M, Budka H, Hainfellner J. Pleomorphic xanthoastrocytoma with anaplastic features presenting without GFAP immunoreactivity: implications for differential diagnosis. Neuropathology. 2005;25:241-6.

12. Zang YS, Dai C, Xu X, Cai X, Wang G, Wei J, Wu A, Sun W, Jiao S, Xu Q. Comprehensive analysis of potential immunotherapy genomic biomarkers in 1000 Chinese patients with cancer. Cancer Med. 2019;8:4699-708.

13. Tekkok IH, Sav A. Anaplastic pleomorphic xanthoastrocytomas. Review of the literature with reference to malignancy potential. Pediatr Neurosurg. 2004;40:171-81.

14. Vu TM, Liubinas SV, Gonzales M, Drummond KJ. Malignant potential of pleomorphic xanthoastrocytoma. J Clin Neurosci. 2012;19:12-20.

15. Okazaki T, Kageji T, Matsuzaki K, Horiguchi H, Hirose T, Watanabe H, Ohnishi T, Nagahiro S. Primary anaplastic pleomorphic xanthoastrocytoma with widespread neuroaxis dissemination at diagnosisa pediatric case report and review of the literature. J Neurooncol. 2009;94:431-7.

16. Gallo P, Cecchi PC, Locatelli F, Rizzo P, Ghimenton C, Gerosa M, Pinna G. Pleomorphic xanthoastrocytoma: long-term results of surgical treatment and analysis of prognostic factors. Br $\mathrm{J}$ Neurosurg. 2013;27:759-64. 
17. Gil-Gouveia R, Cristino N, Farias JP, Trindade A, Ruivo NS, Pimentel J. Pleomorphic xanthoastrocytoma of the cerebellum: illustrated review. Acta Neurochir (Wien). 2004;146:1241-4.

18. Watanabe N, Ishikawa E, Kohzuki H, Sakamoto N, Zaboronok A, Matsuda M, Shibuya M, Matsumura A. Malignant transformation of pleomorphic xanthoastrocytoma and differential diagnosis: case report. BMC Neurol. 2020;20:21.

19. Yamada SM, Murakami H, Tomita Y, Nakane M, Shibui S, Takahashi M, Kawamoto M. Glioblastoma multiforme versus pleomorphic xanthoastrocytoma with anaplastic features in the pathological diagnosis: a case report. Diagn Pathol. 2016;11:65.

20. Alexandrescu S, Korshunov A, Lai SH, Dabiri S, Patil S, Li R, Shih CS, Bonnin JM, Baker JA, Du E, et al. Epithelioid Glioblastomas and Anaplastic Epithelioid Pleomorphic Xanthoastrocytomas-Same Entity or First Cousins? Brain Pathol. 2016;26:215-23.

21. Tanaka S, Nakada M, Nobusawa S, Suzuki SO, Sabit H, Miyashita K, Hayashi Y. Epithelioid glioblastoma arising from pleomorphic xanthoastrocytoma with the BRAF V600E mutation. Brain Tumor Pathol. 2014;31:172-6.

22. Wang J, Liu Z, Cui Y, Liu Y, Fang J, Xu L, He Y, Du J, Su Y, Zou W, et al. Evaluation of EZH2 expression, BRAF V600E mutation, and CDKN2A/B deletions in epithelioid glioblastoma and anaplastic pleomorphic xanthoastrocytoma. J Neurooncol. 2019;144:137-46.

23. Dougherty MJ, Santi M, Brose MS, Ma C, Resnick AC, Sievert AJ, Storm PB, Biegel JA. Activating mutations in BRAF characterize a spectrum of pediatric low-grade gliomas. Neuro Oncol. 2010;12:621-30.

24. Dias-Santagata D, Lam Q, Vernovsky K, Vena N, Lennerz JK, Borger DR, Batchelor TT, Ligon KL, lafrate AJ, Ligon AH, et al. BRAF V600E mutations are common in pleomorphic xanthoastrocytoma: diagnostic and therapeutic implications. PLoS One. 2011;6:e17948.

25. Broniscer A, Tatevossian RG, Sabin ND, Klimo P Jr, Dalton J, Lee R, Gajjar A, Ellison DW. Clinical, radiological, histological and molecular characteristics of paediatric epithelioid glioblastoma. Neuropathol Appl Neurobiol. 2014;40:327-36.

26. Luo W, Li C, Huang J, Chen X, Ma Y, Zeng L. Clinicopathologic changes and molecular finding of epithelioid pleomorphic xanthoastrocytoma: a case report. Int J Clin Exp Pathol. 2018;11:5144-8.

27. Sugita Y, Shigemori M, Okamoto K, Morimatsu M, Arakawa M, Nakayama K. Clinicopathological study of pleomorphic xanthoastrocytoma: correlation between histological features and prognosis. Pathol Int. 2000;50:703-8.

28. Martinez R, Carmona FJ, Vizoso M, Rohde V, Kirsch M, Schackert G, Ropero S, Paulus W, Barrantes A, Gomez A, Esteller M. DNA methylation alterations in grade II- and anaplastic pleomorphic xanthoastrocytoma. BMC Cancer. 2014;14:213.

29. Hirose T, Ishizawa K, Sugiyama K, Kageji T, Ueki K, Kannuki S. Pleomorphic xanthoastrocytoma: a comparative pathological study between conventional and anaplastic types. Histopathology. 2008;52:183-93. 
30. Marton E, Feletti A, Orvieto E, Longatti P. Malignant progression in pleomorphic xanthoastrocytoma: personal experience and review of the literature. J Neurol Sci. 2007;252:144-53.

\section{Tables}

Due to technical limitations, table 1 and 2 is only available as a download in the Supplemental Files section.

\section{Figures}

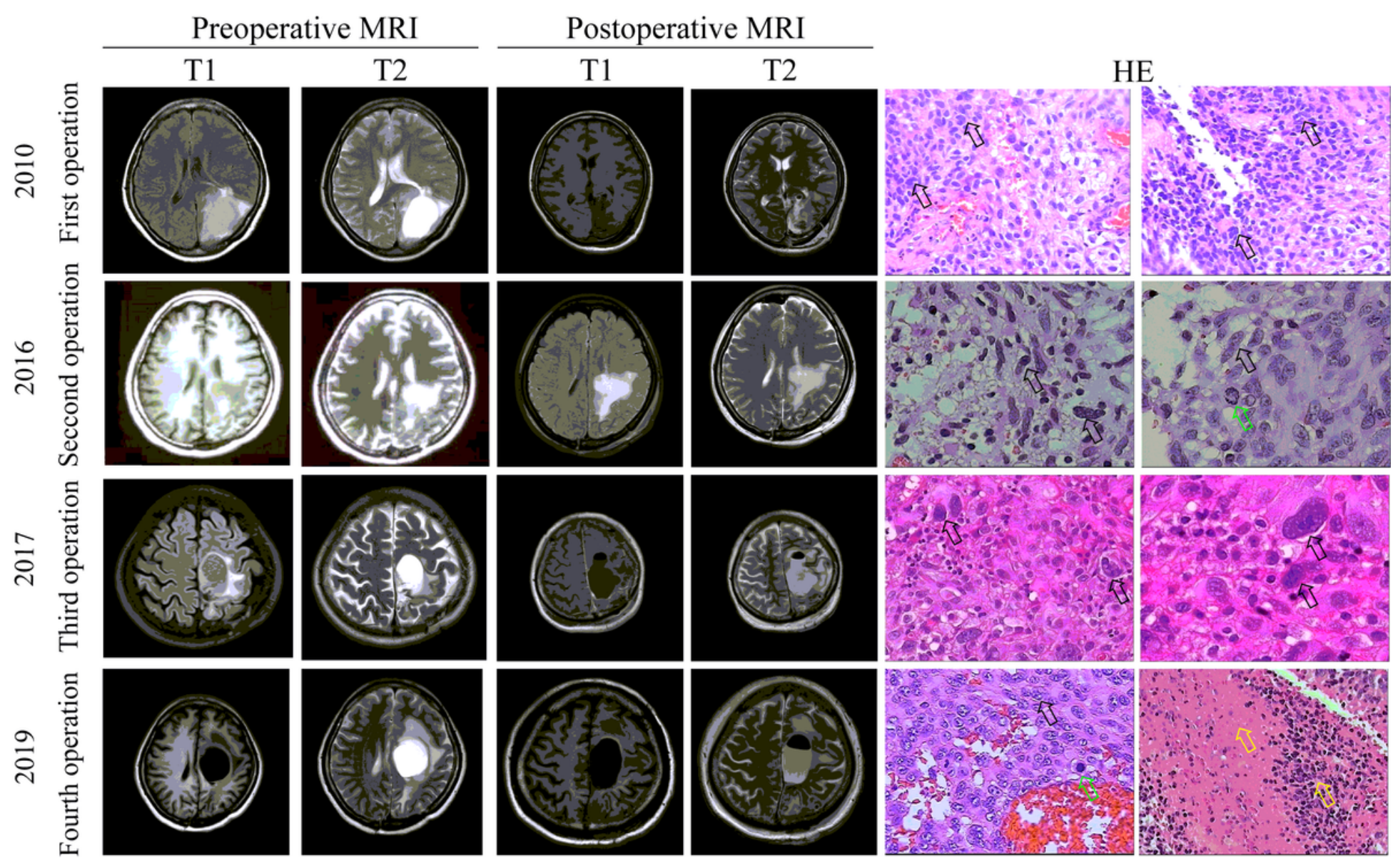

Figure 1

Magnetic resonance imaging (MRI) and haematoxylin-eosin staining (H\&E) of the four surgeries. Preoperative MRI on the left and postoperative MRI on the right. 


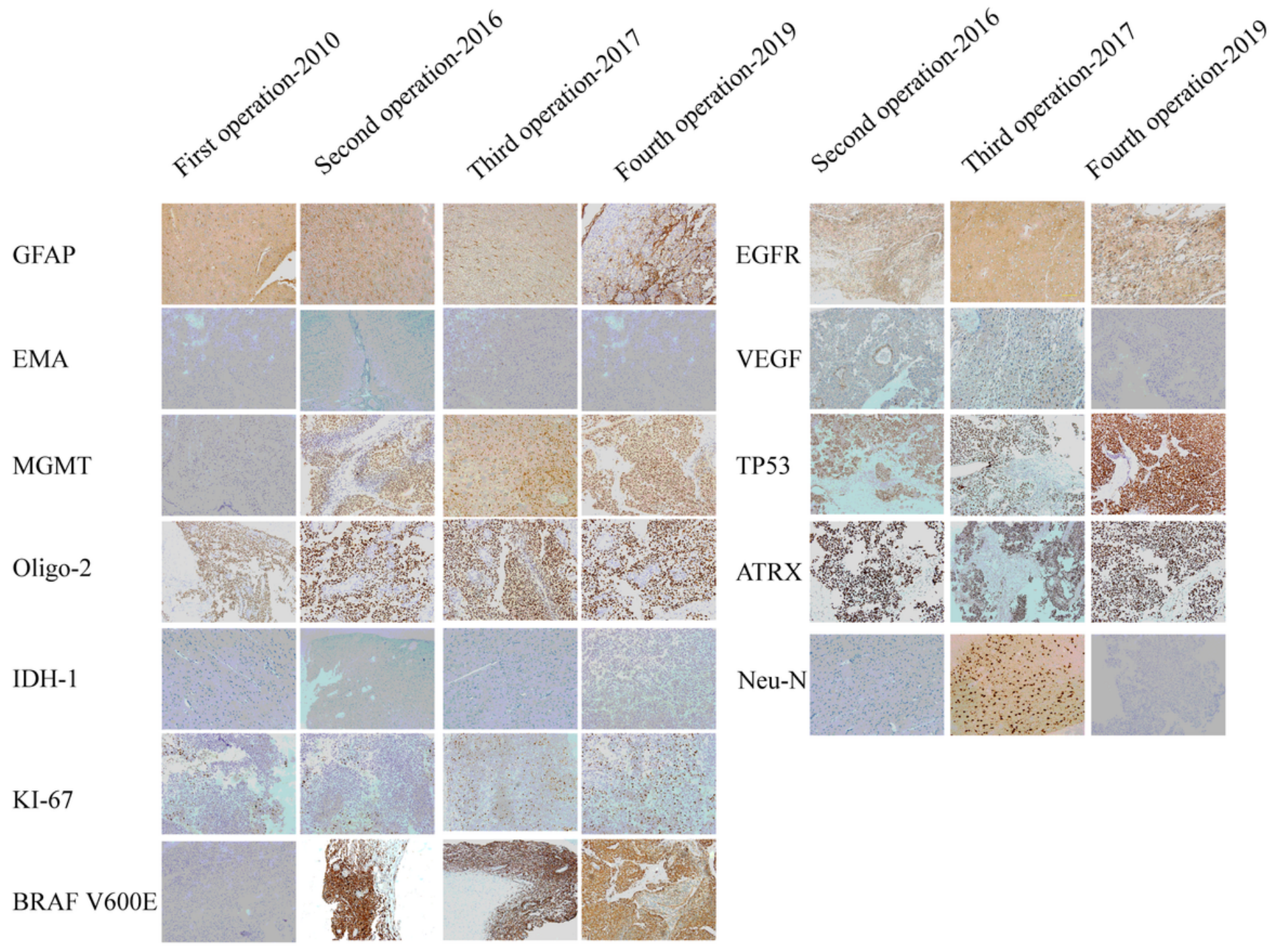

Figure 2

The tumour tissue immunohistochemical features of the four postoperative $(A)$ and the last three postoperative (B) A-PXA (the tumour tissues from the 2010, 2016, and 2017 resections) and E-GBM (the tumour tissues from the 2019 resection) areas in a single case. 


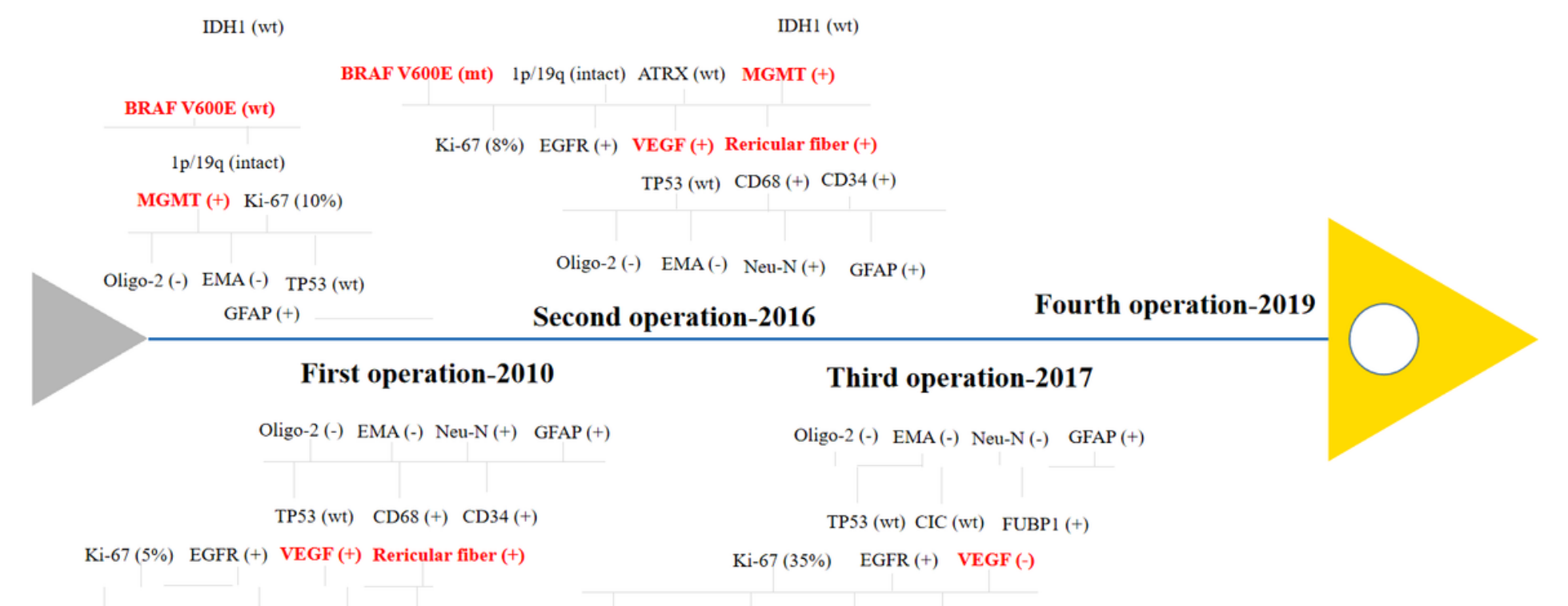

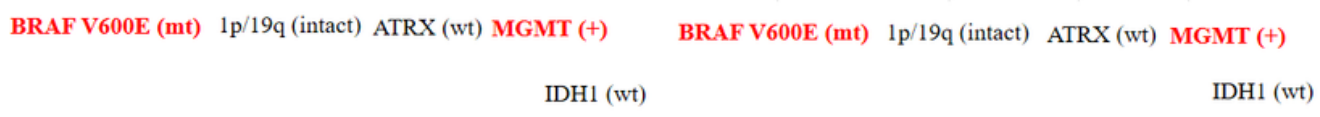

\section{Figure 3}

Fishbone diagram of the molecular characteristics of E-GBM from four postoperative tumours.

\section{Supplementary Files}

This is a list of supplementary files associated with this preprint. Click to download.

- TABLE1.xIsx

- TABLE2.xIsx

- FigureS1.tif

- SupplementaryTable1.xIsx

- SupplementaryTable2.xlsx

- SupplementaryTable3revision1.xlsx

- SupplementaryTable4revision2.xlsx 